\title{
Advanced Genetics Editor checklist
}

\author{
Myles Axton ${ }^{1}$ \\ ${ }^{1}$ Genetics \& Genomics Next Editors
}

June 19, 2022

Editors may return to authors and peer reviewers their assessment of improvements in metadata, data access or reporting transparency that might improve the impact of the article by enabling reproducible research. In some cases, these improvements might need to be made as a precondition for peer review.

This checklist incorporates community ideas for good research practice:

https://www.cos.io/initiatives/top-guidelines

\section{Citation Standards}

1. All data sets and code are cited in the text as References

2. Persistent URI or DOI for data sets and code

3. Citation in Reference section contains author/title/year/URI

\section{Data, Code, and Materials Transparency}

1. Are the following present and sufficient to independently reproduce all claimed results?

\section{For reused public datasets:}

1. data URIs, program code, statistics scripts

\section{For new data and resources}

1. New data available at a trusted digital repository OR all available in paper

2. Variables, parameters, treatment conditions, and observation (number)

3. Full procedures for collection, preprocessing, cleaning, or generating the data

4. Code, scripts, codebooks, algorithms (URI or all in paper)

5. Research materials (description and source) and procedures necessary to conduct an independent replication of the research.

\section{Exceptions for ethical or legal restrictions to reproducible research}

1. Conditions on the dataset or materials that restrict researcher access and use

2. Access protocol for data or materials

3. Access to software and other documentation

4. Indicate all data and materials without the above constraints

\section{Resources available at a community-endorsed public repository}

1. Authors request delay of access until publication 
2. Do the editors identify any factors that might delay peer review/ delay acceptance for publication/ require editorial explanation upon publication /need expression of editorial concern / risk author might need to retract because of reproducibility concerns?

\section{Design and Analysis Transparency}

1. Authors declare reporting is in accordance with community standards listed on http://www. equatornetwork.org/ or https://fairsharing.org

2. Editors recommend the authors check the following community standards and report their results accordingly

\section{Replication}

Since we do not believe that even the best peer reviewed experimental design can overcome sources of variation among studies, the policy of the journal is to encourage submission of experimental designs that contain their own replication. We also encourage studies replicating or powered to replicate work published in this journal.

1. Report contains both hypothesis generation and replication in separate studies

2. Report contains an unreplicated study 